\title{
Double diffusive MHD Casson fluid flow in a non-Darcy porous medium with Newtonian heating and thermo-diffusion effects
}

\author{
Gauri Shanker Seth ${ }^{1}$, Rajan Kumar $^{1}$, Rajat Tripathi ${ }^{2 *}$, Arnab Bhattacharyya ${ }^{1}$ \\ ${ }^{1}$ Department of Applied Mathematics, IIT (ISM) Dhanbad 826004, India \\ ${ }^{2}$ Department of Mathematics, NIT Jamshedpur 831014, India
}

Corresponding Author Email: rajat17mnnit@gmail.com

https://doi.org/10.18280/ijht.360446

Received: 2 April 2018

Accepted: 6 September 2018

\section{Keywords:}

Casson fluid, magnetic field, thermal radiation, viscous and joule dissipations, Soret effect

\begin{abstract}
The present study tends to investigate unsteady MHD flow of a Casson fluid near a vertical oscillating plate through a non-Darcy porous medium. The impact of Joule heating, viscous dissipation, thermo-diffusion and Newtonian heating are taken into consideration. Incorporating dimensionless variables and parameters, governing non-dimensional equations are solved by implicit finite difference technique of Crank-Nicolson type. Numerical simulation for the fluid velocity, fluid temperature and species concentration are carried out for a range of values of regulatory flow parameters that characterize the physics of the flow graphically whereas skin friction coefficient, Nusselt number and Sherwood number for the several values of the emerging flow parameters at the plate are presented in tabular form. One of the significant findings of this analysis include that an intensification in the Newtonian heating effect causes a downfall in the rate of heat transfer at the plate whereas another important outcome of the present study is that the concentration of species will gradually increase when we consider higher order chemical reactions, but the incremental effect will almost extinguish after a certain level. The present investigation may have bearings on several engineering processes such as glass blowing, paper production, extrusion of plastic sheet, annealing and tinning of copper wire, spinning of fibers and continuous casting of metals.
\end{abstract}

\section{INTRODUCTION}

The non-Newtonian fluids possess the property of nonlinear relationship between the shear rate and the shear stress unlike the Newtonian fluids which have a very limited application. This model of Newtonian fluid is unable to describe various facts noticed for the fluids involved in nature and other technological use such as soap, paints, emulsions, polymer solutions and so on. Nowadays, nonNewtonian fluid mechanics is throwing a special challenge to mathematicians, physicists and engineers. Rheological properties of non-Newtonian fluids are defined by their constitutive equations. There is a popular non-Newtonian fluid, proposed by Casson [1] for the forecast of flow characteristic of pigment-oil suspension and the model is known as Casson model. Casson fluid belongs to the class of pseudo plastic fluids which is also a type of shear thinning fluid. Non-Newtonian fluids depend on shear rate history. The shear thinning fluids are more viscous than Newtonian fluids at low shear rates while at high shear rates they are less viscous. So, the study of Casson fluid has found outspread applications in various chemical, pharmaceutical and cosmetic industries such as in the production of several chemicals, oil, gas, syrup, juice, cleanser etc. In the context of fluid mechanics, the study of Casson fluid flow was investigated by several scientists, engineers, mathematicians and researchers depending upon different situations. Studies based on Casson fluid flow are due to Khalid et al. [2], Kataria and Patel [3], Hussanan et al. [4] and Seth et al. [5].
The consequence of thermal radiation finds important applications in various scientific and industrial processes such as electrical power generation, furnace design, glass production, design of fins etc. Keeping in mind its significance, Makinde [6] described the behavior of transient MHD flow along a vertical plate of a thermally radiating fluid. Das et al. [7] have researched hydromagnetic flow of a Casson fluid near a vertical oscillating plate. Some other research works in this context are due to Seth et al. [8], Ali et al. [9], Singh and Kumar [10], etc.

It may be worthy to mention that when the considered fluid is sufficiently viscous, the consequence of viscous dissipation becomes very much important in heat transfer analysis. Significant applications of this effect can be found in food processing, instrumentation, tribology, lubrication, polymer manufacturing etc. Thus, keeping in mind such a fruitful impact of viscous dissipation, several leading research works have been carried out. Recently, the effect of viscous dissipation in case of a chemically reactive mixed convection MHD Casson nanofluid flow over a permeable stretching sheet was explored by Ibrahim et al. [11]. Apart from viscous dissipation, a volumetric heat source viz. Joule heating, also appears into the scenario in case of hydromagnetic flows. In particular, mutual influence of Joule heating and viscous dissipation finds applications in heattreated materials traveling between a wind-up roll or materials produced by extrusion and feed roll. Mahatha et al. [12] utilized Spectral Relaxation Method (SRM) to analyze the two dimension MHD boundary layer Newtonian 
nanofluid flow over stretching sheet considering both Joule and viscous dissipations whereas Hayat et al. [13] discussed the effects of viscous and Joule dissipation including Newtonian heating of Williamson fluid past over the stretching sheet. Singh and Kumar [14] examined the impact of thermal radiation on mixed convection flow of a micropolar fluid with heat generation and viscous dissipation. Some of the relevant research investigations are by Yih [15], Abo-Eldahab and El Aziz [16], Jaber [17], Singh and Kumar [18] and Seth et al. [19].

However, in all the above mentioned research investigations, the effect of thermo-diffusion has been generally neglected. But, when a flux in concentration is induced due to gradient in temperature, this effect becomes more significant. The study of Soret effect finds several important applications in metal casting industry, refinement of crude oil etc. In the space science, NASA has created a laser spectroscopy instrument called the SCOF/ FACET interferometer that can measure Soret phenomena which can be used to study thermodynamic effects in the space. Eckert and Drake [20] discussed the consequence of Soret effect in the cases concerning isotope separation and in the mixing of gasses having very light molecular weight $\left(\mathrm{He}, \mathrm{H}_{2}\right)$ and of medium molecular weight (air, $\mathrm{N}_{2}$ ). Seth et al. [21] explored the Soret and Dufour effects in natural convection flow over an inclined stretching sheet. Jha and Singh [22] examined the effect of thermo-diffusion on transient free convection hydromagnetic flow along an impulsively started infinite vertical plate. Other remarkable research works are due to Reddy and Rao [23], Seth et al. [24] and Kataria and Patel [25].

Most of the research studies are generally based on the problems of steady case governed by Darcy model. Darcy's law is valid for viscous flow and if Reynolds number is less than unity for any flow. Darcy model is not acceptable for the porous media which have large pore radius because the inertial impact of porous medium is ignored by Darcy model. Keeping in view, the importance of such studies Kaviany [26] investigated natural convection flow in non-Darcy porous medium within concentric horizontal cylinders. Yang et al. [27] have analyzed numerically flow through nonDarcy porous medium using iterative MAC (Marker and Cell) and chorin pressure iterative technique. Related noticeable studies are due to Mahmoud [28] and Olanrewaju [29].

The study of mass transfer flow taking into account the chemical reaction has found numerous important applications in various hydrometallurgical and chemical industries such as exothermic chemical reaction, food processing, production of glassware and ceramics, undergoing endothermic and catalytic chemical reactors etc. There are two major categories of chemical reactions, viz. homogeneous and heterogeneous chemical reactions. Some fruitful research works in this context are due to Refs. [30-34].

The current work intents to analyze the influence of viscous dissipation, thermo-diffusion and Joule heating on a transient MHD double diffusive flow of a Casson fluid along an oscillating vertical flat plate which is embedded in a nonDarcy porous medium. Darcy-Forchheimer Model is considered for non-Darcy porous medium which is actually an upgradation of the original Darcy law by adding the quadratic inertial term. In order to find the numerical solutions an implicit finite difference scheme of CrankNicolson type is implemented. Numerical findings are well demonstrated either by graphs or in tabular form under the action of various regulatory flow parameters.

\section{FLOW ANALYSIS}

Consider transient hydromagnetic flow of an electrically conducting, heat radiating, incompressible and chemically reactive Casson fluid along a vertical oscillating flat plate embedded in a non-Darcy porous medium under the influence of thermo-diffusion, Joule heating and viscous dissipation. A cartesian coordinate system is selected to represent the flow formation of the problem. The oscillating plate is in upward direction along the $x^{\prime}$-axis, the alignment of $y^{\prime}$-axis is perpendicular to the plane of the plate and $z^{\prime}$ axis is chosen normally to $x^{\prime} y^{\prime}$-plane. A magnetic field of uniform strength $B_{0}$ is applied transversely to the motion of the plate. Initially, there is no movement of both the fluid and the plate. At time $t^{\prime} \leq 0$, the fluid is maintained at uniform species concentration $C_{\infty}^{\prime}$ and uniform temperature $T_{\infty}^{\prime}$. At time $t^{\prime}>0$, the plate begins to oscillate in $x^{\prime} z^{\prime}$-plane with the velocity $u^{\prime}=U_{0} \cos \left(\omega^{\prime} t^{\prime}\right)$, where $\omega^{\prime} t^{\prime}$ and $U_{0}$ denote phase angle and the amplitude of the plate oscillations respectively. The heat transfer characteristics of the present problem is governed by Newtonian heating condition in which heat transfer from the oscillating plate with a finite heat capacity, is directly proportional to plate temperature $T^{\prime}$. Moreover, species concentration is elevated from $C_{\infty}^{\prime}$ to $C_{w}^{\prime}$. A homogenous chemical reaction of order $n$ is assumed to occur at a constant rate $K_{r}^{\prime}$ between the fluid and the dispersing species. The magnetic field induced by the movement of the liquid is considered to be small compared to the applied one and can therefore be ignored. Since the plate has an infinite extent in $z$ and $x^{\prime}$ directions, all the governing variables depends on $t^{\prime}$ and $y^{\prime}$ except pressure. The schematic theme of the model is illustrated in Figure 1.

The rheological equation for the state of the Cauchy stress tensor for the isotropic and incompressible Casson fluid flow can be written in the form [1]

$\tau_{i j}=\left\{\begin{array}{l}2\left(\mu_{B}+\frac{P_{y}}{\sqrt{2 \phi}}\right) \mathrm{e}_{i j}, \quad \phi>\phi_{c}, \\ 2\left(\mu_{B}+\frac{P_{y}}{\sqrt{2 \phi_{c}}}\right) \mathrm{e}_{i j}, \quad \phi<\phi_{c},\end{array}\right.$

where $P_{y}$ denotes the yield stress, $\phi$ is the product of the component of deformation rate with itself, namely, $\phi=$ $e_{i j} e_{i j}, e_{i j}$ indicates the $(i, j)^{\text {th }}$ component of the deformation rate, $\phi_{c}$ is the critical value of $\phi$ based on the non-Newtonian model, $\tau_{i j}$ is the stress tensor of the fluid, and $\mu_{B}$ is the plastic dynamic viscosity of the Casson fluid. From (1) we obtain (for $\phi<\phi_{c}$ )

$\tau_{i j}=2 \mu_{B}\left(1+\frac{1}{\alpha}\right) e_{i j}$,

where $\alpha=\frac{\mu_{B} \sqrt{2 \pi_{c}}}{P_{y}}$ denotes the Casson fluid parameter. The nature of non-Newtonian fluid vanishes and it behaves as Newtonian fluid when $\alpha \rightarrow \infty$. 


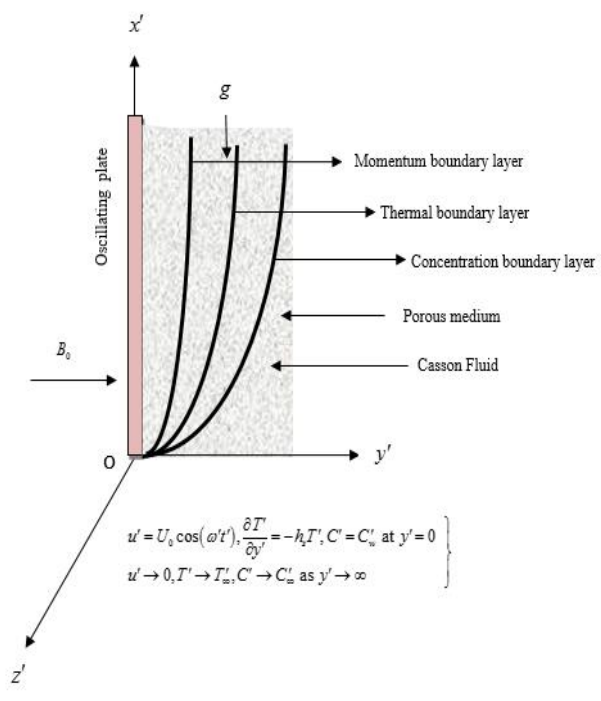

Figure 1. Schematic theme of the model

Under the above assumptions, the governing equations of momentum, energy and concentration of the fluid flow, are given in the form $[7,25,35]$

$$
\begin{aligned}
\frac{\partial u^{\prime}}{\partial t^{\prime}} & =v\left(1+\frac{1}{\alpha}\right) \frac{\partial^{2} u^{\prime}}{\partial y^{\prime 2}}-\left(\frac{\sigma B_{0}^{2}}{\rho}+\frac{v}{K_{p}^{\prime}}\left(1+\frac{1}{\alpha}\right)\right) u^{\prime} \\
& +g \beta_{T}\left(T^{\prime}-T_{\infty}^{\prime}\right)+g \beta_{C}\left(C^{\prime}-C_{\infty}^{\prime}\right)-\frac{F}{K_{p}^{\prime}} u^{\prime 2},
\end{aligned}
$$

$$
\begin{aligned}
\frac{\partial T^{\prime}}{\partial t^{\prime}} & =\frac{k}{\rho c_{p}} \frac{\partial^{2} T^{\prime}}{\partial y^{\prime 2}}-\frac{1}{\rho c_{p}} \frac{\partial q_{r}^{\prime}}{\partial y^{\prime}}+\frac{v}{c_{p}}\left(1+\frac{1}{\alpha}\right)\left(\frac{\partial u^{\prime}}{\partial y^{\prime}}\right)^{2} \\
& +\frac{\sigma B_{0}^{2}}{\rho c_{p}} u^{\prime 2}+\frac{v}{c_{p} K_{p}^{\prime}}\left(1+\frac{1}{\alpha}\right) u^{\prime 2},
\end{aligned}
$$$$
\frac{\partial C^{\prime}}{\partial t^{\prime}}=D \frac{\partial^{2} C^{\prime}}{\partial y^{\prime 2}}-K_{r}^{\prime}\left(C^{\prime}-C_{\infty}^{\prime}\right)^{n}+D_{C T} \frac{\partial^{2} T^{\prime}}{\partial y^{\prime 2}}
$$

The appropriate initial and boundary conditions for the governing equations are:

$u^{\prime}=0, T^{\prime}=T_{\infty}^{\prime}, C^{\prime}=C_{\infty}^{\prime}$ for all $y^{\prime} \geq 0$ and $t^{\prime} \leq 0$

$u^{\prime}=U_{0} \cos \left(\omega^{\prime} t^{\prime}\right), \frac{\partial T^{\prime}}{\partial y^{\prime}}=-h_{s} T^{\prime}, C^{\prime}=C_{w}^{\prime}$ at $y^{\prime}=0$ for $t^{\prime}>0$

$u^{\prime} \rightarrow 0, T^{\prime} \rightarrow T_{\infty}^{\prime}, C^{\prime} \rightarrow C_{\infty}^{\prime}$ as $y^{\prime} \rightarrow \infty \quad$ for $t^{\prime}>0$

where $h_{s}$ is the heat transfer coefficient.

The radiative flux vector $q_{r}^{\prime}$ under Rosseland approximation is as follows:

$q_{r}^{\prime}=-\frac{4 \sigma^{*}}{3 k^{*}} \frac{\partial T^{\prime 4}}{\partial y^{\prime}}$,

where $\sigma^{*}$ and $k^{*}$ denote Stefan-Boltzmann constant and Rosseland mean absorption coefficient respectively. Assuming that the difference in temperature within the flow is small enough, $T^{\prime 4}$ becomes linear function of temperature, by carrying out Taylor series expansion of $T^{\prime 4}$ about the free stream temperature $T_{\infty}^{\prime}$ and ignoring the terms beyond the first order. Thus

$T^{\prime 4} \cong 4 T_{\infty}^{\prime 3} T^{\prime}-3 T_{\infty}^{\prime 4}$

On the use of Eqs. (7) and (8), Eq. (4) becomes

$\frac{\partial T^{\prime}}{\partial t^{\prime}}=\frac{k}{\rho c_{p}} \frac{\partial^{2} T^{\prime}}{\partial y^{\prime 2}}+\frac{1}{\rho c_{p}} \frac{16 \sigma^{*} T_{\infty}^{\prime 3}}{3 k^{*}} \frac{\partial^{2} T^{\prime}}{\partial y^{\prime 2}}+\frac{v}{c_{p}}\left(1+\frac{1}{\alpha}\right)\left(\frac{\partial u^{\prime}}{\partial y^{\prime}}\right)^{2}$

$+\frac{\sigma B_{0}^{2}}{\rho c_{p}} u^{\prime 2}+\frac{v}{c_{p} K_{p}^{\prime}}\left(1+\frac{1}{\alpha}\right) u^{\prime 2}$.

To convert dimensional Eqs. (3), (5) and (9) into nondimensional form, following dimensionless parameters and variables are introduced:

$y=\frac{U_{0}}{v} y^{\prime}, \quad u=\frac{u^{\prime}}{U_{0}}, \quad t=\frac{U_{0}^{2}}{v} t^{\prime}, \quad R=\frac{\sigma B_{0}^{2} v}{\rho U_{0}^{2}}$,

$C=\frac{C^{\prime}-C_{\infty}^{\prime}}{C_{w}^{\prime}-C_{\infty}^{\prime}}, K_{p}=K_{p}^{\prime} \frac{U_{0}^{2}}{v^{2}}, T=\frac{T^{\prime}-T_{\infty}^{\prime}}{T_{\infty}^{\prime}}$,

$G_{r}=\frac{v g \beta_{T} T_{\infty}^{\prime}}{U_{0}^{3}}, N=\frac{16 \sigma^{*} T_{\infty}^{\prime 3}}{3 k k^{*}}, \omega=\omega^{\prime} \frac{v}{U_{0}^{2}}$,

$G_{c}=\frac{v g \beta_{C}\left(C_{w}^{\prime}-C_{\infty}^{\prime}\right)}{U_{0}^{3}}, K_{r}=\frac{\nu K_{r}^{\prime}\left(C_{w}^{\prime}-C_{\infty}^{\prime}\right)^{n-1}}{U_{0}^{2}}$

$\Gamma=\frac{F v}{K_{p}^{\prime} U_{0}}, \quad P_{r}=\frac{v \rho c_{p}}{k}, E_{c}=\frac{U_{0}^{2}}{c_{p} T_{\infty}^{\prime}}, \quad S_{c}=\frac{v}{D}$ and

$S_{r}=\frac{D_{C T} T_{\infty}^{\prime}}{v\left(C_{w}^{\prime}-C_{\infty}^{\prime}\right)}$

Incorporating dimensionless variables (10) into Eqs. (3), (5) and (9), we obtain the governing non-dimensional equations as:

$\frac{\partial u}{\partial t}=\left(1+\frac{1}{\alpha}\right) \frac{\partial^{2} u}{\partial y^{2}}-\left(R+\frac{1}{K_{p}}\left(1+\frac{1}{\alpha}\right)\right) u+G_{r} T+G_{c} C-\Gamma u^{2}$,

$\frac{\partial T}{\partial t}=\frac{1}{\operatorname{Pr}_{e f f}} \frac{\partial^{2} T}{\partial y^{2}}+E_{c}\left(1+\frac{1}{\alpha}\right)\left(\frac{\partial u}{\partial y}\right)^{2}+E_{c}\left(\frac{1}{K_{p}}\left(1+\frac{1}{\alpha}\right)+R\right) u^{2}$,

$\frac{\partial C}{\partial t}=\frac{1}{S_{c}} \frac{\partial^{2} C}{\partial y^{2}}-K_{r} C^{n}+S_{r} \frac{\partial^{2} T}{\partial y^{2}}$,

The associated initial and boundary conditions are as following:

$u=0, T=0, C=0$ for all $y \geq 0$ and $t \leq 0$

$u=\cos \omega t, \frac{\partial T}{\partial y}=-\gamma(1+T), C=1 \quad$ at $y=0$ for $t>0$ 
$u \rightarrow 0, T \rightarrow 0, C \rightarrow 0$ as $y \rightarrow \infty$ for $\quad t>0$

where $\gamma=h_{s} \frac{v}{U_{0}}$ is the Newtonian heating parameter.

\subsection{Skin friction coefficient, Nusselt number and Sherwod number}

Physical quantities of engineering interests such as skin friction coefficient $\tau$, Nusselt number $N_{u}$ [36] and Sherwood number $S_{h}$ are given by

$\tau=\frac{\tau_{w}}{\rho U_{0}^{2}}, \quad N_{u}=\frac{v q_{w}}{U_{0} k\left(T^{\prime}-T_{\infty}^{\prime}\right)}$ and

$S_{h}=\frac{v q_{m}}{U_{0} D\left(C_{w}^{\prime}-C_{\infty}^{\prime}\right)}$,

where $\tau_{w}, q_{w}$ and $q_{m}$ are, respectively, the plate surface shear stress, heat flux from the plate surface and mass flux from the plate, which are given by

$\tau_{w}=-\left.\mu_{B}\left(1+\frac{1}{\alpha}\right) \frac{\partial u^{\prime}}{\partial y^{\prime}}\right|_{y^{\prime}=0}$,

$q_{w}=-\left.k \frac{\partial T^{\prime}}{\partial y^{\prime}}\right|_{y^{\prime}=0}+\left(q_{r}^{\prime}\right)_{y^{\prime}=0}$ and

$q_{m}=-\left.D \frac{\partial C^{\prime}}{\partial y^{\prime}}\right|_{y^{\prime}=0}$

Now, using non-dimensional variables and parameters given by Eq. (10) we obtain skin friction coefficient, Nusselt number and Sherwood number in dimensionless form as

$\tau=-\left.\left(1+\frac{1}{\alpha}\right) \frac{\partial u}{\partial y}\right|_{y=0}$,

$N_{u}=\gamma(1+N)\left[1+\frac{1}{T(0, t)}\right]$ and

$S_{h}=-\left.\frac{\partial C}{\partial y}\right|_{y=0}$.

\section{METHOD OF SOLUTION}

Equations (11) - (13), being coupled nonlinear partial differential equations, restrict us to obtain analytical or exact solutions. So we take the help of numerical methods in order to solve these equations. Finite difference method is one of the most frequently used numerical techniques, which is comparatively simple, precise and effective and has better stability characteristics. We obtain solutions of these equations by implicit scheme of Crank-Nicolson type and another essential feature of this method is the iterative procedure and a tri-diagonal matrix operation. The equivalent finite difference scheme for equations (11) to (13) is as follows:

$$
\begin{aligned}
& \frac{u_{i}^{j+1}-u_{i}^{j}}{\Delta t}=\left(1+\frac{1}{\alpha}\right) \frac{1}{2(\Delta y)^{2}}\left[u_{i-1}^{j}-2 u_{i}^{j}+u_{i+1}^{j}\right. \\
&\left.+u_{i-1}^{j+1}-2 u_{i}^{j+1}+u_{i+1}^{j+1}\right] \\
&\left(R+\frac{1}{K_{p}}\left(1+\frac{1}{\alpha}\right)\right)\left(\frac{u_{i}^{j}+u_{i}^{j+1}}{2}\right) \\
&+\frac{G_{r}}{2}\left(T_{i}^{j}+T_{i}^{j+1}\right)+\frac{G_{c}}{2}\left(C_{i}^{j}+C_{i}^{j+1}\right)-\Gamma\left(u_{i}^{j}\right)^{2}, \\
& \frac{T_{i}^{j+1}-T_{i}^{j}}{\Delta t}= \frac{(1+N)}{P_{r}} \frac{1}{2(\Delta y)^{2}}\left[T_{i-1}^{j}-2 T_{i}^{j}+T_{i+1}^{j}\right. \\
&\left.+T_{i-1}^{j+1}-2 T_{i}^{j+1}+T_{i+1}^{j+1}\right] \\
&+E_{c}\left(1+\frac{1}{\alpha}\right)\left(\frac{\mathrm{u}_{i+1}^{j}-\mathrm{u}_{i}^{j}}{\Delta y}\right)^{2}+E_{c}\left(R+\frac{1}{K_{p}}\left(1+\frac{1}{\alpha}\right)\right)\left(\mathrm{u}_{i}^{j}\right)^{2}, \\
&+\frac{C_{i}^{j+1}-C_{i}^{j}}{\Delta t}=\frac{1}{S_{c}} \frac{1}{2(\Delta y)^{2}}\left[C_{i-1}^{j}-2 C_{i}^{j}+C_{i+1}^{j}\right. \\
&\left.+\frac{S_{r}}{2(\Delta y)^{2}}\left[T_{i-1}^{j}-2 C_{i}^{j+1}+C_{i+1}^{j+1}\right]-K_{r}\left(C_{i}^{j}\right)^{n}+T_{i+1}^{j}+T_{i-1}^{j+1}-2 T_{i}^{j+1}+T_{i+1}^{j+1}\right],
\end{aligned}
$$

The associated initial and boundary conditions take the following form:

$$
\begin{aligned}
& u_{i}^{0}=0, T_{i}^{0}=0, C_{i}^{0}=0 \text { for all } i \\
& u_{0}^{j}=\cos \omega t, T_{1}^{j}=(1-\gamma \Delta y) T_{0}^{j}-\gamma \Delta y, C_{0}^{j}=1 \text { for all } j \\
& u_{M}^{j}=0, T_{M}^{j}=0, C_{M}^{j}=0
\end{aligned}
$$

The region considered here is in the form of a rectangular grid where grid lines are parallel to the coordinate axes with spacing and $\Delta y$ in time and space directions respectively. The grid points are specified by $y_{i}=$ $i \Delta y, i=1,2,3, \ldots, M-1$ and $t_{j}=j \Delta t, j=1,2,3, \ldots, N$. The spatial nodes on the $j^{\text {th }}$ time grid form the $j^{\text {th }}$ level. For computation purpose, the physical domain is converted into computational domain bounded by the lines $y=0$ and $y_{\max }=7$, where $y_{\infty}$ corresponds to $y \rightarrow \infty$ which is enough to satisfy the asymptotic free stream. After experiment with several sets of grid sizes, the grid size in ${ }^{y}$-direction is taken as $\Delta y=h=0.025$ and time step $\Delta t=0.01$. The complete solution of discretized equations (18) to (20) proceed as follows:

1. Knowing the values of $C, T$ and $u$ at all points of the grid at a time $t=j$ from the initial condition, we find $T$ and $C$ at time $t=j+1$ using the previous level of time ' $j$ ' calculated as follows via solving tridiagonal system of equations as explained by Carnahan et al [37].

2. Knowing the values of $T$ and $C$ at times $t=j$ and $t=$ $j+1$ and $u$ at time $t=j$, the values of $u$ at $t=j+1$ time level are calculated in similar manner. This process is repeated to obtain a solution to the desired time $t$.

The Crank-Nicolson technique has a truncation error of $O\left\{(\Delta y)^{2}+(\Delta t)^{2}\right\}$, that tends to zero as $\Delta y$ and $\Delta t$ tend to zero. This method is unconditionally stable [38]. 
Compatibility and stability together provide a convergence scheme.

\subsection{Grid independence analysis}

In order to obtain accuracy, the grid independence analysis has been carried out. The developed code has been executed taking different mesh (element) sizes. For this we have calculated the values of skin friction coefficient $\tau$ under the influence of Casson parameter $\alpha$ and permeability parameter $K_{p}$ taking mesh sizes $h=0.10, h=0.05, h=0.025$ and $h=$ 0.01 and keeping the value of $\Delta t$ fixed at $\Delta t=0.01$ and the results are presented in Table 1 . From Table 1 it is found that when we make a reduction in the mesh size by $50 \%$ from the initial mesh size $h=0.10$, the numerical values of skin friction coefficient become similar upto second decimal place with the previous one. In the next step, when the mesh size is again lowered to $h=0.025$, a resemblance in the values of skin friction coefficient is noticed upto fourth decimal place. Further, if we consider the mesh size $h=0.01$, we observe that the numerical values of skin friction coefficient become convergent upto sixth decimal place to those obtained at $h=$ 0.025 . Hence, we can conclude that at $h=0.025$ the numerical solutions become stable and turn out to be independent of the size of the mesh upto sixth decimal place.

Table 1. Comparison of values of skin friction coefficient $\boldsymbol{\tau}$ for different mesh sizes

\begin{tabular}{|c|c|c|c|c|c|}
\hline$\alpha$ & $\boldsymbol{K}_{\boldsymbol{p}}$ & $\boldsymbol{h = 0 . 1 0}$ & $\boldsymbol{h = 0 . 0 5}$ & $\boldsymbol{h = 0 . 0 2 5}$ & $\boldsymbol{h}=\mathbf{0 . 0 1}$ \\
\hline \multirow{2}{*}{0.2} & 0.2 & 0.62739541 & 0.62695498 & 0.62691364 & 0.62691358 \\
\cline { 2 - 6 } & 0.5 & 0.16620469 & 0.16589831 & 0.16587992 & 0.16587984 \\
\hline \multirow{2}{*}{0.4} & 0.2 & 0.77927836 & 0.77908712 & 0.77906543 & 0.77906541 \\
\cline { 2 - 6 } & 0.5 & 0.31924667 & 0.31894793 & 0.31891657 & 0.31891654 \\
\hline
\end{tabular}

\subsection{Validation of numerical result}

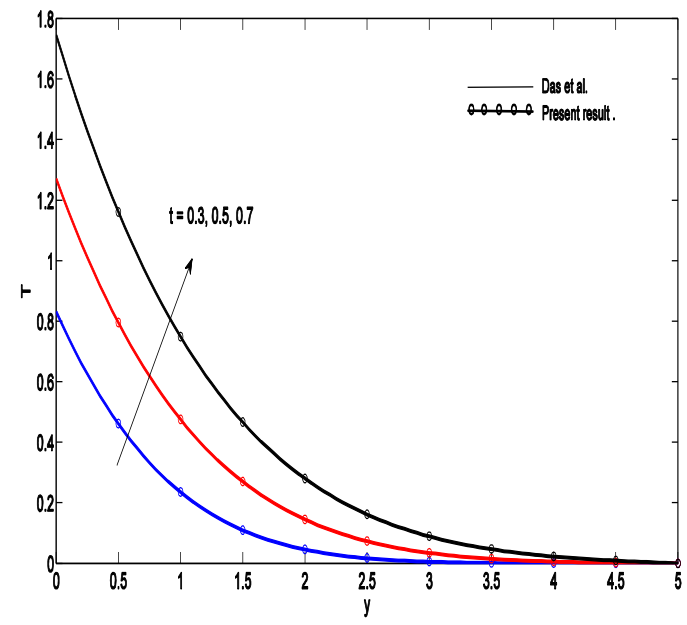

Figure 2. Comparison of temperature profile with Das et al. [7] for different values of $t$

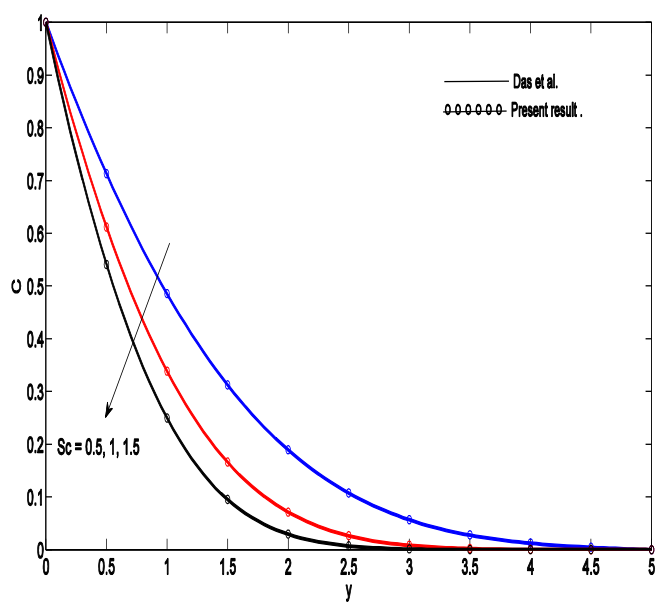

Figure 3. Comparison of concentration profile with Das et al. [7] for different values of $S_{c}$
To validate our developed code, the numerical solutions are compared from the used scheme with the exact solutions found by Das et al. [7] for several values of $t$ and $S_{c}$ by taking $\Gamma=E_{c}=S_{r}=K_{p}{ }^{-1}=0$ and $n=1$. The exact solutions obtained by Das et al. [7] are presented by solid straight lines while the numerical solutions obtained by our developed code of are presented by solid-circled lines. As one can see from Figs. 2 and 3 that there is an excellent agreement within the exact solutions obtained by Das et al. [7] and the numerical solutions computed in the present study.

\section{RESULTS AND DISCUSSION}

To obtain the physical vision of the considered model, comprehensive numerical calculations have been performed. The graphical representations for fluid velocity, fluid temperature and species concentration are shown in Figs. 4 to 18 for different values of regulatory flow parameters that characterize the physics of the flow. The quantities of engineering interests i.e. skin friction coefficient, Nusselt number and Sherwood number for different values of regulatory flow parameters are presented in Tables 2 to 4 . The numerical calculations are performed for all the governing flow parameters by selecting default values $\alpha=$ $0.4, K_{p}=0.2, G_{c}=2, G_{r}=3, \Gamma=0.2, P r_{e f f}=3, E_{c}=0.1$, $t=0.7, \gamma=0.5, S_{r}=0.5, K_{r}=0.5, n=1$ and $\omega t=\pi / 4$, until otherwise specified particularly.

Figures 4 to 6 elucidate the three-dimensional plot for the velocity, temperature and concentration profiles respectively. These profiles are drawn due to the variation in time. As one can observe from Figure 4 that velocity profiles are drawn for a maximum time period of $t=0.7$. This is because with the gradual increment in the time we can notice that the velocity profile tends to become convergent. From Figure 5 it can be perceived that curves representing temperature of the fluid start from different points. This trend is because of Newtonian heating condition. In the same way from Figure 6 we can conclude that species concentration of the fluid is also maximum when $t=0$.7and then after a certain time the solutions start to converge. 


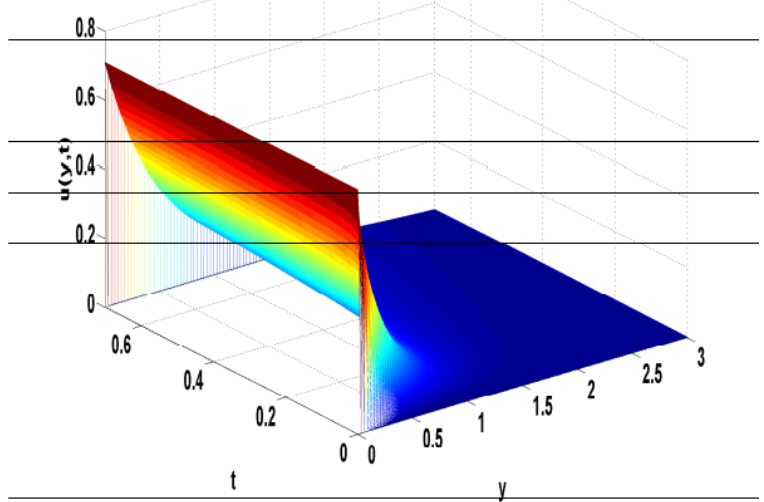

Figure 4. Three-dimensional plot of velocity profile for variation of $t$.

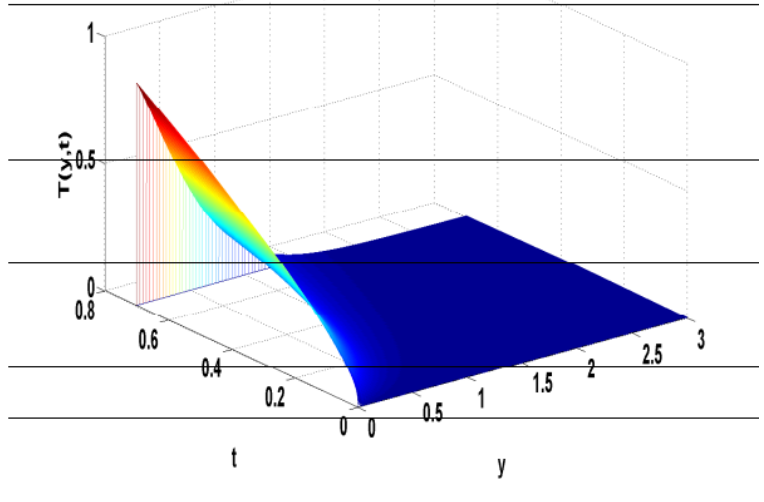

Figure 5. Three-dimensional plot of temperature profile for variation of $t$.

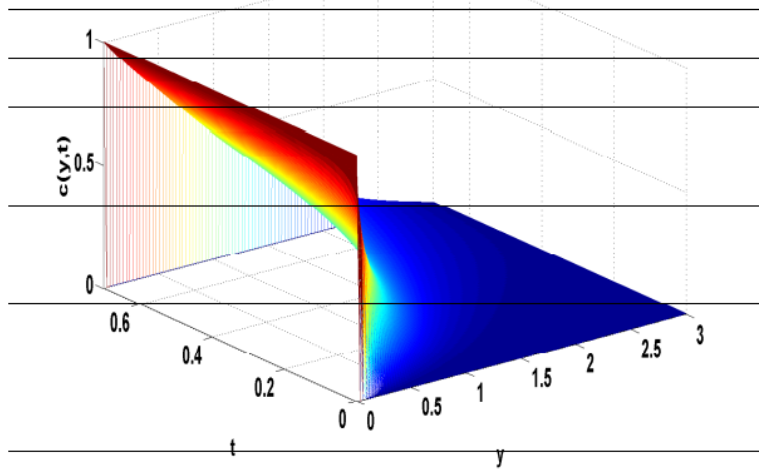

Figure 6. Three-dimensional plot of concentration profile for variation of $t$.

The behavior of fluid velocity under the influence of regulatory flow parameters such as permeability parameter $K_{p}$, Casson parameter $\alpha$, thermal Grashof number $G_{r}$, solutal Grashof number $G_{c}$, and local inertia parameter (Forchheimer number) $\Gamma$ are depicted in Figs. 4 to 8 , respectively. Figure 4 portrays the impact of Casson parameter $\alpha$ on the velocity field. It is perceived that velocity of the fluid decreases with the increment in $\alpha$. This phenomena can be attributed to the fact that a rise in the value of $\alpha$ means increment in the plastic dynamic viscosity of the Casson fluid $\mu_{B}$, thereby producing a resistance to the fluid flow and as a result, velocity of the fluid gets reduced. Figure 5 describes the effect of permeability parameter $K_{p}$, on the velocity field. As we see that the velocity is getting increased with the enhancement in permeability parameter. The physics behind this fact can be justified, as an increment in permeability means bigger pore sizes within the porous medium. Thus an increasing fluid velocity is observed. The effects of thermal and solutal Grashof numbers $G_{r}$ and $G_{c}$ are demonstrated in Figs. 6 and 7 respectively. The trend of the graphs in Figs. 6 and 7 indicate that velocity increases as we increase values of both $G_{r}$ and $G_{c}$. Physically, this is acceptable because $G_{r}$ is defined as the ratio of thermal buoyancy force to viscous force and $G_{c}$ as the ratio of solutal buoyancy force to viscous force. So, growing values of $G_{r}$ and $G_{c}$ make thermal and solutal buoyancy forces stronger, which consequently accelerate the velocity of the fluid. Figure 8 displays the impact of Forchheimer parameter $\Gamma$ on the velocity profile. When $\Gamma=0$, it represents a Darcy porous media. An enhancement in the Forchheimer parameter reduces the fluid velocity due to increase in the quadratic drag.

In order to highlight the impact of regulatory flow parameters: Eckert number $E_{c}$, effective Prandtl number [39] $P r_{e f f}=\frac{P_{r}}{1+N}$, Newtonian heating parameter $\gamma$ and time $t$ on fluid temperature $T$, we have plotted the graphs of fluid temperature versus these parameters, which are shown in Figs. 9 to 12. Figure 9 elucidates that the increasing value of effective Prandtl number $\operatorname{Pr}_{\text {eff }}$ reduces the temperature of the fluid. Since $P r_{e f f}=\operatorname{Pr} /(1+N), P r_{e f f}$ increases when $P_{r} \quad$ increases whereas $P r_{e f f}$ decreases when $N$ increases. An enhancement in $P_{r}$ weakens the thermal diffusivity of the fluid, hence the fluid temperature is getting decreased. One can perceive from Figure 9 that fluid temperature increases on decreasing $\operatorname{Pr}_{\text {eff }}$. Thus we arrive to conclusion that fluid temperature is enhanced due to strengthening of thermal radiation. A gradual elevation in fluid temperature $T$ is noted with the enhancement in Eckert number, as observed from Figure 10. Eckert number is expressed as the relationship between the flow of kinetic energy and enthalpy difference of boundary layer. $E_{c}$ signifies the conversion of kinetic energy into internal energy, generated by the work done against the viscous fluid stresses. Therefore, higher viscous dissipative heat results in enhanced temperature of the fluid. Figure 11 portrays the impact of Newtonian heating parameter $\gamma$ on the temperature profile. Here one can observe that Newtonian heating parameter tends to increase the fluid temperature. As $\gamma \rightarrow \infty$, the Newtonian heating condition becomes prescribed wall temperature case. Figure 12 shows that with the progress of time $t$, fluid temperature gets enhanced.

The graphical results are described for concentration profiles $C$ against Soret number $S_{r}$, chemical reaction parameter $K_{r}$, order of chemical reaction nand time $t$. Figure 13 reflects thermo-diffusion effect on species concentration. One can observe that Soret number $S_{r}$ uplifts the concentration of the species. Physical clarification of this fact is that a mass flux from lower to higher species concentration 
driven by temperature gradient is produced by Soret effect and that is why the diffusive species with higher values of Soret number corresponds to thicker concentration boundary layer. Figure 14 implies that a rise in the positive values of chemical reaction parameter $K_{r}$ results in a significant downfall of species concentration. The central reason behind this fact is that positive $K_{r}$ represents the destructive kind of chemical reaction. Thus an enhancement in positive values of $K_{r}$ decreases the species concentration with a healthier rate which in consequence produces a retarding effect on concentration distribution. It is evident from Figs. 15 and 16 that species concentration is an increasing function of both $n$ and $t$. This suggests that concentration of the species will be gradually increasing as we consider higher order chemical reactions but the incremental effect almost die out after a certain level. Likewise, with the progress of time, an improvement in species concentration is observed. To analyze the nature of physical quantities of interests viz. Sherwood number $S_{h}$, skin friction coefficient $\tau$ and Nusselt number $N_{u}$ with respect to various governing parameters, Tables 2 to 4 have been prepared. Table 2 displays the numerical values of skin friction coefficient under the influence of $\alpha, K_{p}, G_{r}, G_{c}$ and $\Gamma$. It is evident from Table 2 that there is an increment in the values of skin friction coefficient on increasing $\alpha$ and $\Gamma$ whereas a reverse trend for skin friction coefficient is noted for $K_{p}, G_{r}$ and $G_{c}$. This implies that Casson parameter and Forchheimer number have the tendency to elevate the skin friction coefficient whereas permeability parameter, thermal and solutal Grashof numbers tend to lower this physical quantity. Variation of Nusselt number $N_{u}$ are displayed in Table 3 , for $P r_{e f f}, t, \gamma$ and $E_{c}$. It can be inferred from Table 3 , that the rate of heat transfer at the plate is enhanced for increasing value of $P r_{\text {eff }}$ while an absolutely opposite trend is noticed for $t, \gamma$ and $E_{c}$. This signifies that Prandtl number and thermal radiation tend to rise the heat transfer rate at the plate while a downfall in this physical quantity occurs with the increment in Newtonian heating parameter, Eckert number and time. Table 4 portrays the behavior of Sherwood number with respect to $S_{r}, K_{r}$ and $t$. From Table 4, one can notice that Sherwood number increases on increasing $K_{r}$ whereas an adverse effect is noticed in case of $S_{r}$ and $t$. This suggests that chemical reaction parameter intensifies the rate of mass transfer at the plate while a decrement in the values of $S_{h}$ is perceived on increment in Soret number and time.

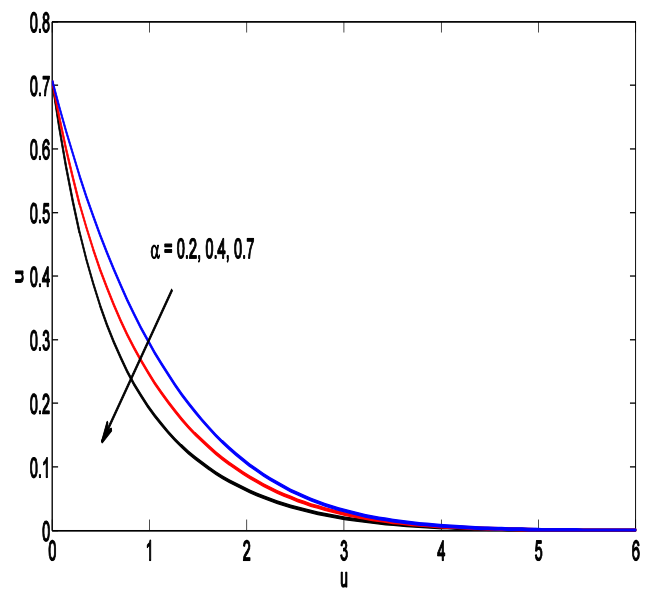

Figure 7. Influence of $\alpha$ on fluid velocity

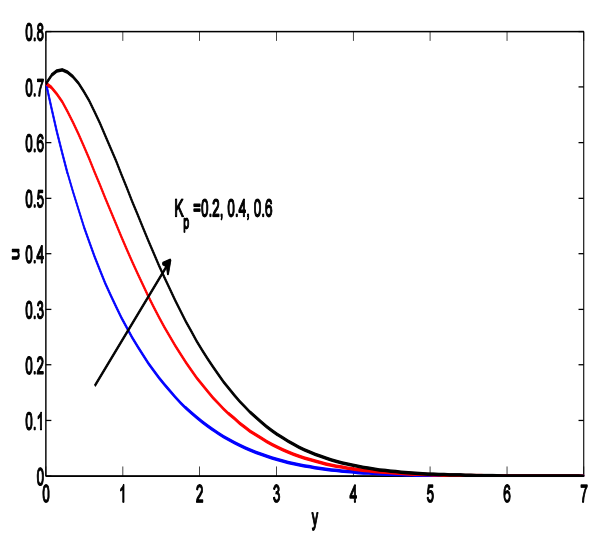

Figure 8. Influence of $K_{p}$ on fluid velocity

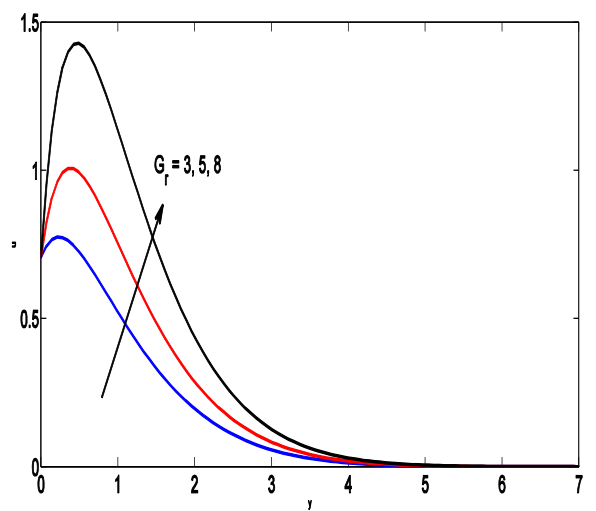

Figure 9. Influence of $G_{r}$ on fluid velocity

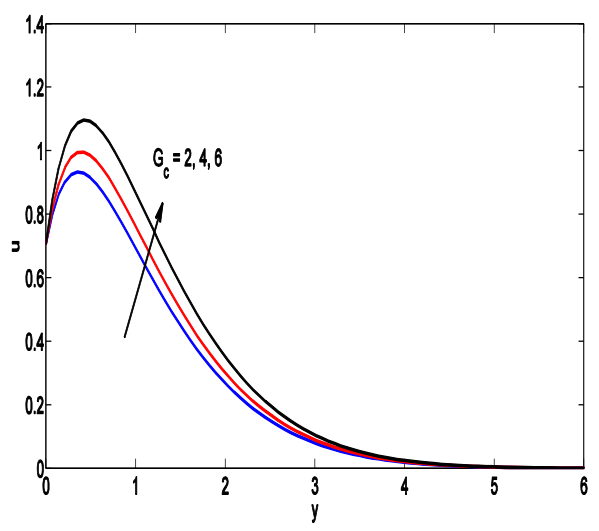

Figure 10. Influence of $G_{c}$ on fluid velocity

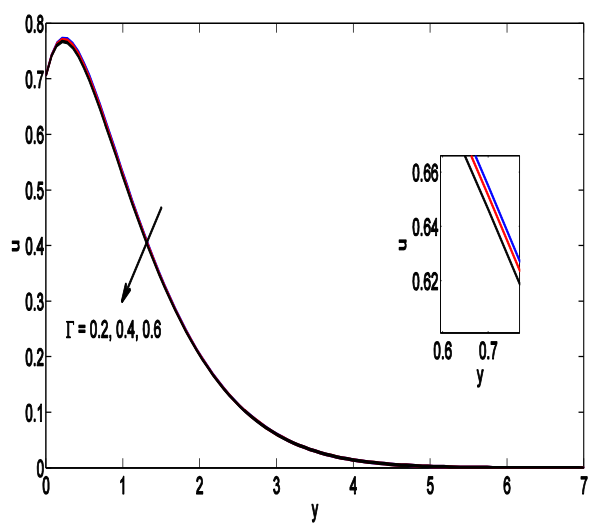

Figure 11. Influence of $\Gamma$ on fluid velocity 


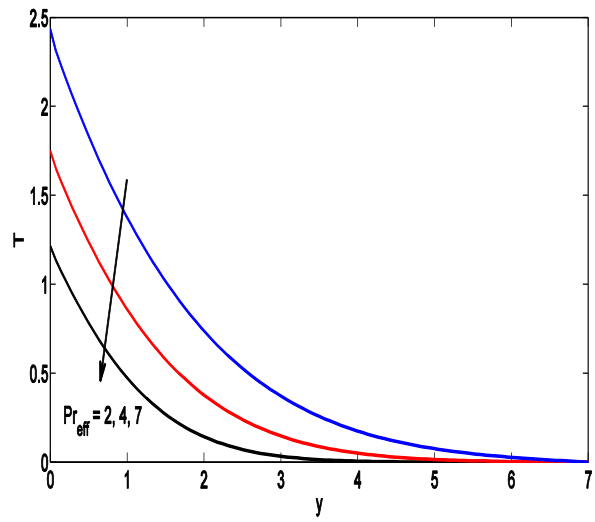

Figure 12. Influence of $P r_{e f f}$ on fluid temperature

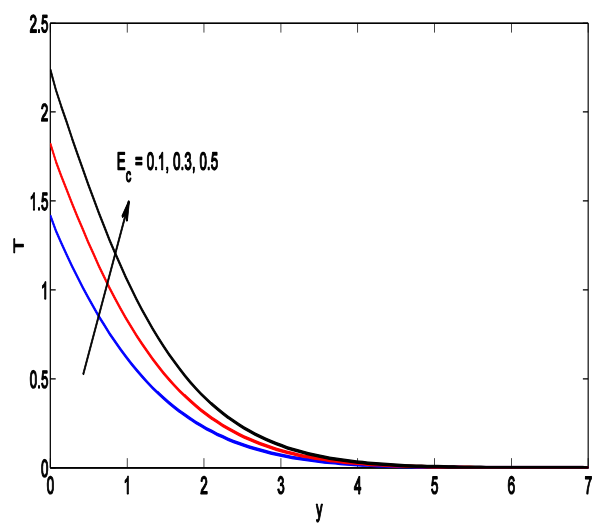

Figure 13. Influence of $E_{c}$ on fluid temperature

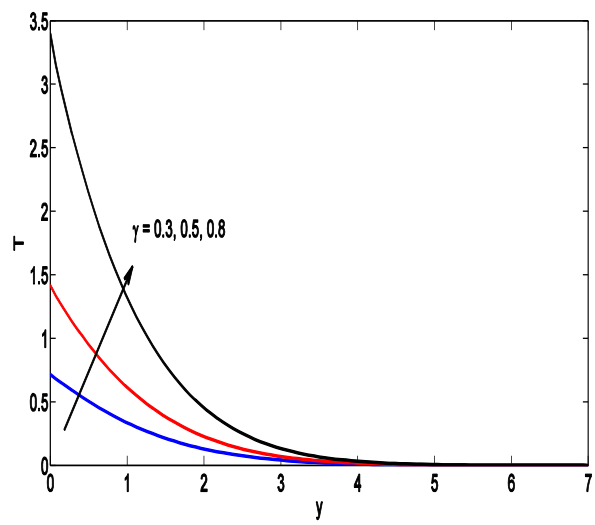

Figure 14. Influence of $\gamma$ on fluid temperature

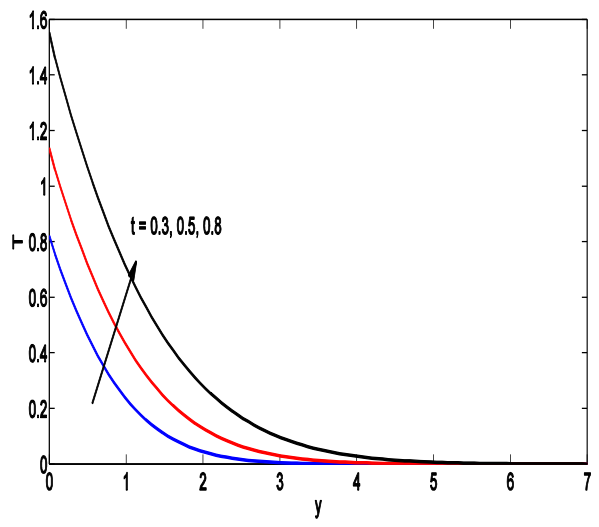

Figure 15. Influence of $t$ on fluid temperature

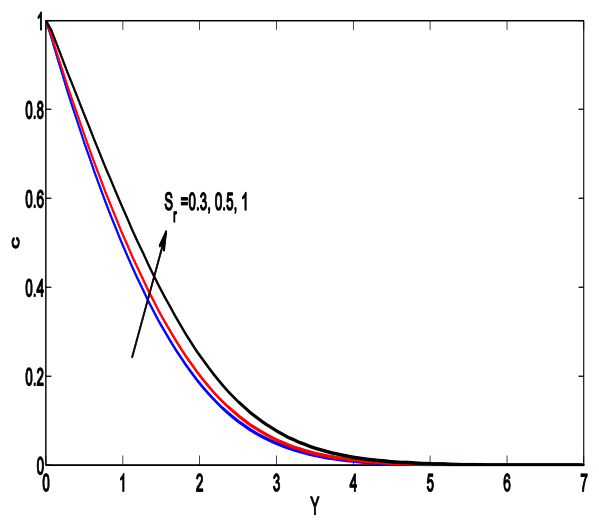

Figure 16. Influence of $S_{r}$ on fluid concentration

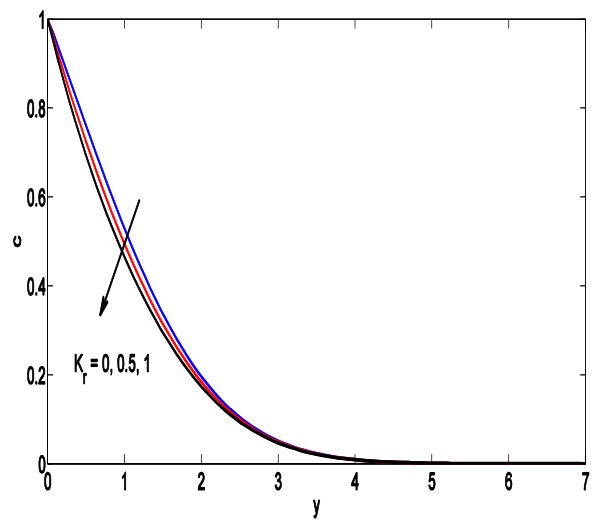

Figure 17. Influence of $K_{r}$ on fluid concentration

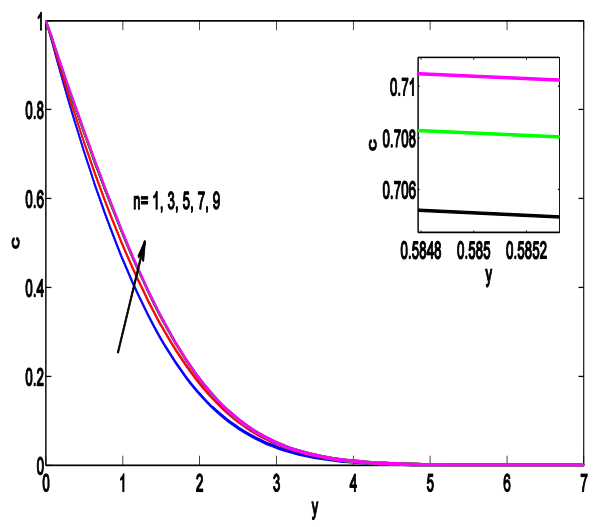

Figure 18. Influence of $n$ on fluid concentration

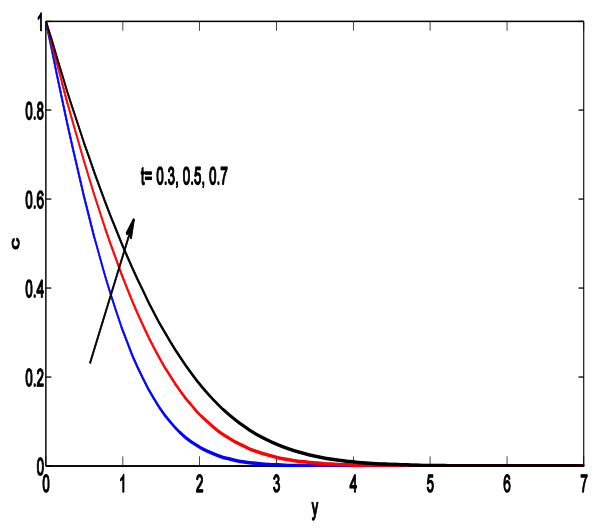

Figure 19. Influence of $t$ on fluid concentration 
Table 2. Skin friction coefficient $\tau$ for differents values of regulatory flow parameter

\begin{tabular}{|c|c|c|c|c|c|}
\hline$\alpha$ & $\boldsymbol{K}_{\boldsymbol{p}}$ & $\boldsymbol{G}_{\boldsymbol{r}}$ & $\boldsymbol{G}_{\boldsymbol{c}}$ & $\Gamma$ & $\tau$ \\
\hline 0.2 & & & & & 3.88704208 \\
0.4 & 0.2 & 3 & 2 & 0.2 & 4.01464129 \\
0.6 & & & & & 4.19094113 \\
\hline & 0.1 & & & & 5.65336650 \\
0.4 & 0.2 & 3 & 2 & 0.2 & 4.01464129 \\
& 0.5 & & & & 2.41863134 \\
\hline & & 3 & & & 4.01464129 \\
0.4 & 0.2 & 5 & 2 & 0.2 & 3.72198836 \\
& & 8 & & & 3.27423418 \\
\hline & & & 2 & & 4.01464129 \\
0.4 & 0.2 & 3 & 4 & 0.2 & 3.47584359 \\
& & & 6 & & 2.93494351 \\
\hline & & & & 0.2 & 4.01464129 \\
0.4 & 0.2 & 3 & 2 & 0.4 & 4.02568064 \\
& & & & 0.6 & 4.03667082 \\
\hline
\end{tabular}

Table 3. Nusselt number $N_{u}$ for various values of regulatory flow parameters

\begin{tabular}{|c|c|c|c|c|}
\hline$t$ & $\gamma$ & $\operatorname{Pr}_{\text {eff }}$ & $\boldsymbol{E}_{\boldsymbol{c}}$ & $\boldsymbol{N}_{u}$ \\
\hline 0.3 & & & & 3.22837666 \\
0.5 & 0.5 & 3 & 0.1 & 2.60946516 \\
0.7 & & & & 2.29138066 \\
\hline & 0.1 & & & 3.39849574 \\
0.7 & 0.2 & 3 & 0.1 & 3.02131142 \\
& 0.5 & & & 2.29138066 \\
\hline & & 1 & & 2.03641453 \\
0.7 & 0.5 & 3 & 0.1 & 2.29138066 \\
& & 5 & & 2.33208074 \\
\hline & & & 0.1 & 2.29138066 \\
0.7 & 0.5 & 3 & 0.2 & 1.82913637 \\
& & & 0.5 & 1.39620590 \\
\hline
\end{tabular}

Table 4. Sherwood number $S_{h}$ for differents values of regulatory flow parameters

\begin{tabular}{|c|c|c|c|}
\hline $\boldsymbol{S}_{\boldsymbol{r}}$ & $\boldsymbol{K}_{\boldsymbol{r}}$ & $\boldsymbol{t}$ & $\boldsymbol{S}_{\boldsymbol{h}}$ \\
\hline $\mathbf{0 . 3}$ & & & 0.58390597 \\
$\mathbf{0 . 5}$ & 0.5 & 0.7 & 0.53380575 \\
$\mathbf{1}$ & & & 0.40918457 \\
\hline & $\mathbf{0}$ & & 0.46650980 \\
0.3 & $\mathbf{0 . 5}$ & 0.7 & 0.53380575 \\
& $\mathbf{1}$ & & 0.68794317 \\
\hline & & $\mathbf{0 . 3}$ & 0.84118512 \\
0.3 & 0.5 & $\mathbf{0 . 5}$ & 0.67079925 \\
& & $\mathbf{0 . 7}$ & 0.53380575 \\
\hline
\end{tabular}

\section{CONCLUSIONS}

In the present investigation, the numerical simulation of transient MHD flow of a Casson fluid with the consideration of Joule heating, viscous dissipation and thermo-diffusion near a moving infinite non-conducting oscillating vertical flat plate embedded in a non-Darcy porous medium is performed by implicit finite difference technique of Crank-Nicolson type. Noteworthy outcomes of this investigation are summarized as follows:

- The three-dimensional plot for the velocity, temperature and concentration profiles explain completely to account for a certain level of time when the solution starts to converge.
- Since Casson fluid parameter and local inertia parameter induce a resistance to the fluid flow, the fluid velocity gets lowered with the increment in the values of these parameters. On the other hand, an advancement in thermal and solutal Grashof numbers and permeability parameter lead to enhance the velocity of the fluid throughout the boundary layer region.

- A significant downfall in fluid temperature is observed due to increase in effective Prandtl number whereas Newtonian heating parameter, Eckert number and time cause an elevation in the fluid temperature.

- Species concentration of the fluid is reduced as we increase the values of chemical reaction parameter while an adverse effect is perceived in case of Soret number, order of chemical reaction and time.

- Skin friction coefficient is getting improved on increasing either of Casson parameter and local inertia parameter whereas a reverse trend is followed by permeability parameter, solutal Grashof number and thermal Grashof number.

- Nusselt number $N_{u}$ is enhanced with an increase in effective Prandtl number while a gradual decrement in this physical quantity is noted with increasing the values of Eckert number, Newtonian heating parameter and time.

- Sherwood number $S_{h}$ is getting enhanced with an advancement in chemical reaction parameter whereas this physical quantity is getting diminished as we increase the values of Soret number, and time.

\section{REFERENCES}

[1] Casson N. (1959). A flow equation for the Pigment-oil suspensions of the printing ink type. In: Rheology of Disperse Systems, Pergamon New York. 84-102.

[2] Khalid I, Khan A, Khan, Shafie S. (2015). Unsteady MHD free convection flow of Casson fluid past over an oscillating vertical plate embedded in a porous medium. Engineering Science and Technology, an International Journal 18(3): 309-317. https://doi.org/10.1016/j.jestch.2014.12.006

[3] Kataria HR, Patel HR. (2016). Radiation and chemical reaction effects on MHD Casson fluid flow past an oscillating vertical plate embedded in porous medium. Alexandria Engineering Journal 55: 583-595. https://doi.org/10.1016/j.aej.2016.01.019

[4] Hussanan MZ, Khan SI, Tahar RM. (2017). Heat transfer in magnetohydrodynamic flow of a Casson fluid with porous medium and Newtonian heating. Journal of Nanofluids 6(4): 784-793.

[5] Seth GS, Tripathi R, Mishra MK. (2017). Hydromagnetic thin film flow of a Casson fluid in a non-Darcy porous medium with Joule dissipation and Navier's partial slip. Applied Mathematics and Mechanics (English Edition) 38(11): 1613-1626.

[6] Makinde OD. (2012.). Chemically reacting hydromagnetic unsteady flow of a radiating fluid past a vertical plate with constant heat flux. Zeitschrift für Naturforschunga 67(5): https://doi.org/10.5560/ZNA.2012-0014 
[7] Das M, Mahato R, Nandkeolyar R. (2015). Newtonian heating effect on unsteady hydromagnetic Casson fluid flow past a flat plate with heat and mass transfer. Alexandria Engineering Journal 54(4): 871-879. https://doi.org/10.1016/j.aej.2015.07.007

[8] Seth GS, Bhattacharyya A, Tripathi R. (2017). Effect of hall current on MHD natural convection heat and mass transfer flow of rotating fluid past a vertical plate with ramped wall temperature. Frontiers in Heat and Mass Transfer (FHMT) 9(21): 12.

[9] Ali F, Gohar M, Khan I. (2016). MHD flow of waterbased Brinkman type nanofluid over a vertical plate embedded in a porous medium with variable surface velocity, temperature and concentration. Journal of Molecular Liquids 223: 412-419.

[10] Singh K, Kumar M. (2014). Melting heat transfer in boundary layer stagnation point flow of MHD micropolar fluid towards a stretching / shrinking surface. Jordan Journal of Mechanical and Industrial Engineering 8(6): 403-408.

[11] Ibrahim SM, Lorenzini G, Kumar PV, Raju CSK. (2017). Influence of chemical reaction and heat source on dissipative MHD mixed convection flow of a Casson nanofluid over a nonlinear permeable stretching sheet. International Journal of Heat and Mass Transfer 111: 346-355. https://doi.org/10.1016/j.ijheatmasstransfer.2017.03.097

[12] Mahatha BK, Nandkeolyar R, Mahto GK, Sibanda P. (2016). Dissipative effects in hydromagnetic boundary layer nanofluid flow past a stretching sheet with Newtonian heating. Journal of Applied Fluid Mechanics 9(4): 1977-1989.

[13] Hayat T, Shafiq A, Farooq MA, Alsulam HH, Shehzad SA. (2016). Newtonian and Joule heating effects in twodimensional flow of Williamson fluid. Journal of Applied Fluid Mechanics 9(4): 1969-1975.

[14] Singh K, Kumar M. (2016). Effects of thermal radiation on mixed convection flow of a micro-polar fluid from an unsteady stretching surface with viscous dissipation and heat generation/abs. International Journal of Chemical Engineering http://dx.doi.org/10.1155/2016/8190234

[15] Yih KA. (2000). Viscous and Joule heating effects on non-Darcy MHD natural convection flow over a permeable sphere in porous media with internal heat generation. International Communications in Heat and $\begin{array}{lll}\text { Mass } & \text { Transfer } & \text { 27(4): }\end{array}$ https://doi.org/10.1016/S0735-1933(00)00141-X

[16] Abo-Eldahab EM, El Aziz MA. (2005). Viscous dissipation and Joule heating effects on MHD free convection from a vertical plate with power-law variation in surface temperature in the presence of Hall and ion-slip currents. Applied Mathematical Modelling 29(6): 579-595

[17] Jaber KK. (2014). Effects of viscous dissipation and Joule heating on MHD flow of a fluid with variable properties past a stretching vertical plate. European Scientific Journal 10(33): 383-393.

[18] Singh K, Kumar M. (2015). Effect of viscous dissipation on double stratified MHD free convection in Micro-polar fluid flow in porous media with chemical reaction, heat generation and Ohmic eating. Chemical and Process Engineering Research 31, ISSN 2224-7467, ISSN 2225-0913 (Online).
[19] Seth GS, Kumar R, Bhattacharyya A. (2018). Entropy generation of dissipative flow of carbon nanotubes in rotating frame with Darcy-Forchheimer porous medium. Journal of Molecular Liquids 268: 637-646.

[20] Eckert ERG, Drake RM. (1972). Analysis of Heat and Mass Transfer, 1st Edition, McGraw-Hill.

[21] Seth GS, Tripathi R, Rashidi MM. (2017). Hydromagnetic natural convection flow in a non-Darcy medium with Soret and Dufour effects past an inclined stretching sheet. Journal of Porous Media 20(10): 941960.

[22] Jha BK, Singh AK. (1990). Soret effects on freeconvection and mass transfer flow in the Stokes problem for an infinite vertical plate. Astrophysics and Space Science 173(2): 251-255.

[23] Reddy BP, Rao JA. (2011). Radiation and thermal diffusion effects on an unsteady MHD free convection mass-transfer flow past an infinite vertical porous plate with the Hall current and a heat source. Journal of Engineering Physics and Thermophysics 84(6): 13691378.

[24] Seth GS, Kumbhakar B, Sarkar S. (2015). Soret and Hall effects on unsteady MHD free convection flow of radiating and chemically reactive fluid past a moving vertical plate with ramped temperature in rotating system. International Journal of Engineering Science and Technology 7(2): 94-108.

[25] Kataria HR, Patel HR. (2016). Soret and heat generation effects on MHD Casson fluid flow past an oscillating vertical plate embedded through porous medium. Alexandria Engineering Journal 55(3): 2125-2137.

[26] Kaviany M. (1986). Non Darcian effect on natural convection in porous medium confined between horizontal cylinders. International Journal of Heat and Mass Transfer 29(10): 1513-1519.

[27] Yang D, Yang Y, Costa VAF. (2009). Numerical simulation of non-Darcian flow through a porous medium. Particuology 7(3): 193-198.

[28] Mahmoud MAA. (2009). Heat generation/absorption and viscous dissipation effects on MHD flow of a micropolar fluid over a moving permeable surface embedded in a non-Darcian porous medium. Journal of the Korean Physical Society 54(4): 1526-1531.

[29] Olanrewaju PO. (2012). Effects of internal heat generation on hydromagnetic non-Darcy flow and heat transfer over a stretching sheet in the presence of thermal radiation and Ohmic dissipation. World Applied Science Journal 16: 37-45.

[30] Singh K, Kumar M. (2015). The effect of chemical reaction and double stratification on MHD free convection in micro-polar fluid in heat generation and Ohmic heating. Jordan Journal of Mechanical and Industrial Engineering 9(4): 279-288.

[31] Singh K, Kumar M. (2018). Influence of chemical reaction on MHD boundary layer flow of a micropolar fluid over a wedge with Hall and ion-slip currents. International Journal of Engineering Papers 3(1): 1-9.

[32] Singh K, Kumar M. (2016). Influence of chemical reaction on heat and mass transfer flow of a micropolar fluid over a permeable channel with radiation and heat generation. Journal of Thermodynamics. http://dx.doi.org/10.1155/2016/8190234

[33] Singh K, Pandey A, Kumar M. (2018). Analytical approach to a stagnation point flow and heat transfer of 
a micropolar fluid via a permeable shrinking sheet with slip and convective boundary conditions. Heat Transfer Research.

http://dx.doi.org/10.1615/HeatTransRes.2018024647

[34] Seth GS, Mahto N, Tripathi R, Kumar R. (2018). Freestream-induced unsteady MHD Flow with hall effect over permeable plate in a rotating system. applications of fluid dynamics. Lecture notes in Mechanical Engineering. https://doi.org/10.1007/978-981-10-53290_4

[35] Shit GC, Haldar R, Mandal S. (2017). Entropy generation on MHD flow and convective heat transfer in a porous medium of exponentially stretching surface saturated by nanofluids. Advanced Powder Technology 28(6): 1519-1530.

[36] Narhari M, Nayan MY. (2011). Free convection flow past an impulsively started infinite vertical plate with Newtonian heating in the presence of thermal radiation and mass diffusion. Turkish Journal of Engineering and Environmental Sciences 35: 187-198.

[37] Carnahan B, Luther HA, Wilkes JO. Applied numerical methods. John Wiley \& Sons, New York, 1969.

[38] Potter D. (1973). Computational Physics. Wiley, Hoboken.

[39] Magyari E, Pantokratoras A. (2011). Note on the effect of thermal radiation in the linearized Rosseland approximation on the heat transfer characteristics of various boundary layer flows. International Communications in Heat and Mass Transfer 38(5): 554556.

\section{NOMENCLATURE}

$g \quad$ acceleration due to gravity

$q_{r}^{\prime} \quad$ radiative heat flux

$u^{\prime} \quad$ velocity of the fluid along $x^{\prime}$-axis

$T^{\prime} \quad$ fluid temperature
$\mathrm{K}_{p}^{\prime} \quad$ permeability of porous medium

$K_{r}^{\prime} \quad$ chemical reaction coefficient

$C^{\prime} \quad$ species concentration

$F \quad$ quadratic drag coefficient

$c_{p} \quad$ specific heat at constant pressure

$D_{C T} \quad$ soret diffusivity

$k \quad$ thermal conductivity of the fluid

$D$ molecular mass diffusivity

$E_{c} \quad$ Eckert number

$K_{r} \quad$ chemical reaction parameter

$K_{p} \quad$ permeability parameter

$N \quad$ radiation parameter

$G_{c} \quad$ solutal Grashof number

$G_{r} \quad$ thermal Grashof number

$\mathrm{R}$ magnetic parameter

$\mathrm{S}_{c} \quad$ Schimdt number

$P_{r} \quad$ Prandtl number

$\mathrm{Pr}_{\text {eff }} \quad$ effective Prandtl number

$S_{r} \quad$ Soret number

\section{Greek symbols}

$\sigma \quad$ electrical conductivity

$v \quad$ kinematic coefficient of viscosity

$\rho \quad$ density of the fluid

$\beta_{T} \quad$ thermal expansion coefficient

$\beta_{C} \quad$ coefficient of volumetric expansion

$\Gamma \quad$ Forchheimer number

$\omega$ frequency parameter 